\title{
Preferred Hexoses Influence Long-Term Memory in and Induction of Lactose Catabolism by Streptococcus mutans
}

\author{
Lin Zeng, ${ }^{a}$ Lulu Chen, ${ }^{a, b}$ (D) Robert A. Burne ${ }^{a}$ \\ aDepartment of Oral Biology, University of Florida, College of Dentistry, Gainesville, Florida, USA \\ bState Key Laboratory of Oral Diseases, National Clinical Research Center for Oral Diseases, Department of \\ Pediatric Dentistry, West China Hospital of Stomatology, Sichuan University, Chengdu, Sichuan, China
}

ABSTRACT Bacteria prioritize sugar metabolism via carbohydrate catabolite repression, which regulates global gene expression to optimize the catabolism of preferred substrates. Here, we report an unusual long-term memory effect in certain Streptococcus mutans strains that alters adaptation to growth on lactose after prior exposure to glucose or fructose. In strain GS-5, cells that were first cultured on fructose and then transferred to lactose displayed an exceptionally long lag (>11 h) and slower growth compared to cells first cultured on glucose or cellobiose, which displayed a reduction in lag phase by as much as $10 \mathrm{~h}$. When grown on lactose, mutants lacking the cellobiose-phosphotransferase (PTS) or phospho- $\beta$-glucosidase lost the accelerated growth associated with prior culturing on glucose. The memory effects of glucose or fructose on lactose catabolism were not as profound in strain UA159, but the lag phase was considerably shorter in mutants lacking the glucosePTS EIIMan. Interestingly, when S. mutans was cultivated on lactose, significant quantities of free glucose accumulated in the medium, with higher levels found in the cultures of strains lacking EllMan, glucokinase, or both. Free glucose was also detected in cultures that were utilizing cellobiose or trehalose, albeit at lower levels. Such release of hexoses by $S$. mutans is likely of biological significance as it was found that cells required small amounts of glucose or other preferred carbohydrates to initiate efficient growth on lactose. These findings suggest that $S$. mutans modulates the induction of lactose utilization based on its prior exposure to glucose or fructose, which can be liberated from common disaccharides.

IMPORTANCE Understanding the molecular mechanisms employed by oral bacteria to control sugar metabolism is key to developing novel therapies for management of dental caries and other oral diseases. Lactose is a naturally occurring disaccharide that is abundant in dairy products and commonly ingested by humans. However, for the dental caries pathogen Streptococcus mutans, relatively little is known about the molecular mechanisms that regulate expression of genes required for lactose uptake and catabolism. Two peculiarities of lactose utilization by $S$. mutans are explored here: (i) S. mutans excretes glucose that it cleaves from lactose, and (ii) prior exposure to certain carbohydrates can result in a long-term inability to use lactose. The study begins to shed light on how S. mutans may utilize bet hedging to optimize its persistence and virulence in the human oral cavity.

KEYWORDS Streptococcus mutans, lactose, fructose metabolism, growth arrest, sugar:phosphotransferase system

$\mathrm{D}$ ental caries is a disease caused by metabolic activities of tooth biofilms, driven primarily by the fermentation by microorganisms of carbohydrates to organic acids that dissolve the tooth minerals. The development of caries usually requires ingestion of substantial amounts of dietary carbohydrates over an extended period of
Received 11 April 2018 Accepted 29 April 2018

Accepted manuscript posted online 11 May 2018

Citation Zeng L, Chen L, Burne RA. 2018. Preferred hexoses influence long-term memory in and induction of lactose catabolism by Streptococcus mutans. Appl Environ Microbiol 84:e00864-18. https://doi.org/10 .1128/AEM.00864-18.

Editor Maia Kivisaar, University of Tartu Copyright $\odot 2018$ American Society for Microbiology. All Rights Reserved. Address correspondence to Robert A. Burne, rburne@dental.ufl.edu. 
time. In developed nations, starches, sucrose, high-fructose corn syrups that contain a mixture of glucose and fructose, and lactose are among the most abundant carbohydrates in the human diet. Since feeding in humans is intermittent, the oral microbiota endures a "feast-or-famine" existence (1), wherein oral biofilms persist during fasting periods on nutrients provided in saliva and other oral secretions, end products generated by microorganisms, and microbially produced storage compounds that include intracellular glycogen-like polysaccharides and structurally and compositionally diverse extracellular polysaccharides. The microbiome is generally believed to be carbohydratelimited when the host is fasting. Most bacteria employ carbohydrate catabolite repression (CCR) to prioritize carbohydrate utilization when more than one metabolizable source is present, shifting to less-preferred carbohydrates after preferred sources have been exhausted. The primary effector of CCR in low-G $+C$ Gram-positive bacteria is usually catabolite control protein $(\mathrm{CpPA})$, which differentially regulates a significant portion of the transcriptome in response to certain glycolytic intermediates (2), including fructose-1,6-bisphosphate (F-1,6-bP). The major etiological agent for human dental caries, Streptococcus mutans, is a Gram-positive, lactic acid-generating bacterium capable of metabolizing an array of carbohydrates, even at $\mathrm{pH}$ values as low as 3.8 (3). To persist and cause disease, $S$. mutans must compete with a variety of metabolically similar commensal streptococci and with other caries pathogens. With the recognition that dental caries is a polymicrobial infectious disease, there is a need to improve our understanding of the molecular mechanisms and the effects on bacterial ecology of the prioritization and optimization of carbohydrate utilization by the oral microbiome.

Significant progress has been made in understanding CCR in S. mutans, including the characterization of the $\mathrm{CcpA}$ regulon and its role in regulating central carbon metabolism and virulence gene expression $(4,5)$. S. mutans is unusual, however, in that CcpA does not play a major role in CCR by directly regulating catabolic genes for nonpreferred carbohydrates. Instead, CcpA-independent pathways are the primary routes for managing CCR in this human pathogen. First, a major role in CCR has been demonstrated for the $A B$ domain of the primary glucose/mannose-specific phosphotransferase (PTS) permease EIIMan, encoded by manL, which regulates the expression of the fructanase operon (fruAB), a fructose-PTS operon (levDEFG), the cellobiose-PTS pathway (celA and celRBCD), and the lactose operon (lacABCDFEG) (6-9). A manL mutant of S. mutans UA159 showed significant defects in biofilm formation, acid production, and competence development $(10,11)$. Similarly, a mutant defective in the sucrose-PTS permease ( $s c r A$ ) showed altered expression of the fruAB and levDEFG operons (12). HPr, and in particular the serine-phosphorylated form of the protein $\left(\mathrm{HPr}\right.$-Ser46- $\left.\mathrm{PO}_{4}\right)$, is also a potent effector of CCR for certain catabolic systems in S. mutans, as is the case for $B$. subtilis and other low-G+C Gram-positives.

Due in large part to the widespread adoption of diets rich in refined carbohydrates, fructose and lactose have become increasingly abundant in human foods in recent decades. However, unlike for sucrose, the relative contribution of these and many other sugars to human dental caries and the overall impact on the oral microbiome are not well understood. Fructose is considered a preferred sugar for many oral bacteria and is catabolized by oral streptococci in a manner similar to glucose. Nearly $5 \%$ of the transcriptome of $S$. mutans is differentially expressed ( $>2$-fold change in mRNA levels) in response to fructose compared to cells growing on glucose (13), indicative of a major shift in the physiological state of cells when the two different hexoses are present. Notably, many genes encoding proteins needed for development of genetic competence and for stress tolerance showed enhanced expression in cells growing on fructose. A recent study probing the molecular mechanisms by which fructose affects gene expression in $S$. mutans revealed that FruR regulates expression of a primary fructose-PTS (frul) and the glucose/mannose-PTS operon ( $m a n L M N$ ) in concert with the CcpA protein (14). It was also reported that accumulation of phosphorylated fructose derivatives, particularly in a fruK (1-phosphofructokinase) mutant, adversely affected the ability of the organism to grow on multiple carbohydrates, and that the loss of FruK altered the expression of $\sim 400$ genes. 
Lactose is a $\beta 1,4$-linked disaccharide of glucose and galactose. Catabolism of lactose by bacteria usually requires cleavage of the disaccharide, either inside or outside the cell, or following internalization and phosphorylation by the PTS. Lactose metabolism in $S$. mutans depends on the gene products of the lac operon, which encodes a lactose-specific PTS (lacFE) for transport and phosphorylation of lactose, a phospho- $\beta$ galactosidase (lacG) for cleavage of lactose-6-phosphate (Lac-6-P), and the tagatose pathway $(\operatorname{IaCABCD})$ for metabolism of the galactose-6-phosphate $(\mathrm{Gal}-6-\mathrm{P})(9,15)$ that is released from Lac-6-P. Intracellular glucose, which can be phosphorylated by a glucokinase $(g / k)$ and enter the glycolytic pathway, is also generated. Expression of the lac genes requires activation by Gal-6-P, which derepresses the operon presumably by acting as an allosteric regulator of the Lacl-type regulator LacR. The expression of the lac operon is also subject to negative regulation by the glucose-PTS and is repressed when sufficient levels of glucose are available in the environment (9). When both lactose and glucose are present, $S$. mutans preferentially internalizes and catabolizes glucose (16). After glucose is depleted, cells shift to the use of lactose for growth, with batch-cultured cells exhibiting a classic diauxic growth curve (16). The underlying mechanism for glucose-dependent repression appears to be inducer exclusion, whereby expression of the lac genes is inhibited due to a failure to transport lactose and cleave it to produce the apparent cognate inducer (Gal-6-P); however, other factors that may regulate repression and induction have not yet been elucidated (9). The tagatose pathway is the primary route for catabolism of galactose by $S$. mutans, but there are strain-specific differences that dictate whether galactose is transported by a galactose-specific PTS or the lactose-PTS, with EllMan serving as a secondary loweraffinity transporter for galactose (9).

We posit that the ability of $S$. mutans to coordinate and optimize the utilization of preferred carbohydrates and nonpreferred sources to compete with commensals and throughout the caries process is an essential virulence attribute. To better understand the molecular mechanisms used by S. mutans and related organisms, we examined the transition of $S$. mutans from preferred sugars to nonpreferred sources using lactose as a model. The results from these experiments revealed an unusual memory and growth arrest phenomena, with glucose, fructose, and the PTS regulating these behaviors.

\section{RESULTS AND DISCUSSION}

History of exposure to glucose or fructose affects adaptation to lactose. To investigate how $S$. mutans prioritizes carbohydrate metabolism, we performed a series of growth assays examining the transition from preferred sugars to lactose. Starting from an overnight culture prepared using a modified FMC medium that was constituted with a final concentration of $20 \mathrm{mM}$ glucose or fructose, S. mutans cultures were diluted 1:10 into the same media, subcultured to midexponential phase, and then diluted (1:20) into fresh FMC containing $10 \mathrm{mM}$ lactose. Growth on lactose was monitored using a Bioscreen C for 48 h. S. mutans GS-5 and a number of other strains of S. mutans (10449, LM7, V403, and SMU44) did not display any appreciable growth for several hours (12 to $15 \mathrm{~h}$ for GS-5) after transfer to lactose when the cells were precultured in FMC-fructose, but initiated growth within $2 \mathrm{~h}$ after transfer from FMCglucose to FMC-lactose (Fig. 1A, Table 1, and see Fig. S1A in the supplemental material). Most of these strains (except LM7) also exhibited slower growth and attained lower optical densities (ODs) (see Fig. S1A in the supplemental material) following transfer from FMC-fructose to FMC-lactose compared to cells transferred from FMC-glucose. Similar behaviors were observed under conditions when cells were transferred to a mixture of $5 \mathrm{mM}$ lactose and $5 \mathrm{mM}$ glucose or fructose (see Fig. S2 in the supplemental material), where significantly slower growth was noted from cells precultivated on fructose, when they were transferred onto FMC containing a mixture of glucose and lactose. These results suggest that growth on fructose induced a form of long-term "memory" that prevented cells from initiating growth on lactose. This effect is not a simple manifestation of CCR, since cells transitioned in a much more typical fashion from glucose to lactose, and the effects of precultivation in fructose persisted even 

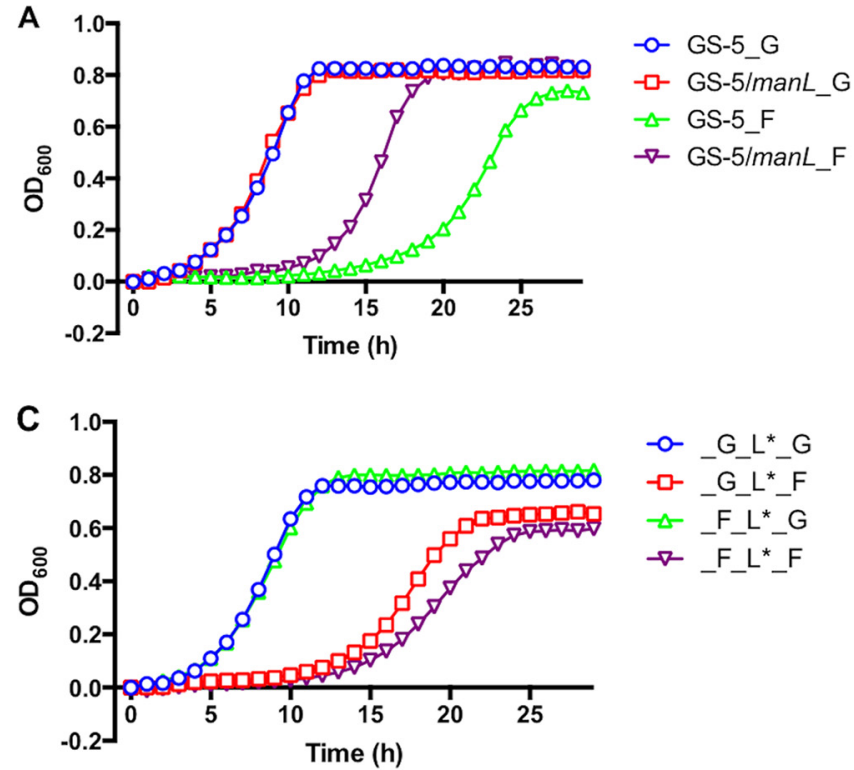

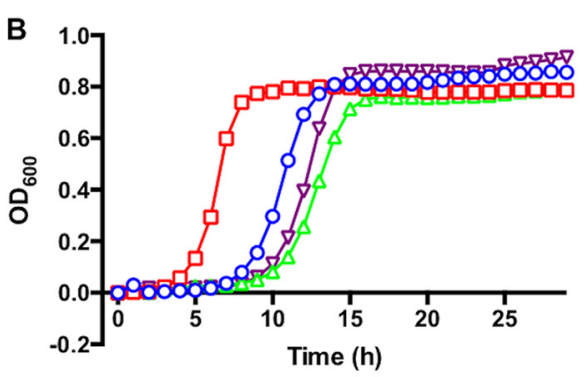

$-0-G_{-} L^{*}-G$
$-\square-G_{-} L^{*} F$
$-\Delta-F_{L^{*}}{ }^{*} G$
$\rightarrow-F_{-} L^{*}{ }^{*} F$
-o- UA159_G
- - UA159/manL G
$\triangle$ UA159_F
$\rightarrow$ UA159/manL_F

FIG 1 Growth of S. mutans on lactose after transferring from glucose or fructose. Wild-type strains GS-5 (A) and UA159 (B) and their otherwise-isogenic mutants deficient in glucose-PTS permease (manL) were first cultivated in FMC containing 20 mM glucose (designated_G) or fructose (_F) until exponential phase and then diluted at a 1:20 ratio into fresh FMC constituted with $10 \mathrm{mM}$ lactose for growth monitoring on a Bioscreen $C$ system. (C) The final cultures from panel A were spread onto $\mathrm{BHI}$ agar plates to form individual colonies, designated_G_L* and_F_L* which were cultivated again on glucose (_G) or fructose (_F) and diluted into FMC containing lactose.

when glucose was used alone, in place of lactose, or added to the FMC-lactose (data not shown and Fig. S2 in the supplemental material).

The results presented above contrasted with what was observed with the commonly utilized laboratory strain UA159 and certain other isolates of S. mutans (UA101, SMU33, SMU21, and OMZ175) (Fig. 1B, Table 1, and see Fig. S1B in the supplemental material). In particular, the times required to initiate growth on lactose following transfer from fructose were much shorter than for the strains described in Fig. S1A in the supplemental material, with no long-term memory being evident. However, the time it took for glucose-grown cells to initiate growth on lactose increased for these strains, except for OMZ175. Glucose is internalized primarily by the glucose/mannose-PTS transporter EIIMan, so the growth behaviors of ElIMan mutants, generated by deleting manL or manLMN, in the UA159 and GS-5 genetic backgrounds were also evaluated. The results (Fig. 1B and Table 1) showed that, compared to the wild-type strains, the EllMan mutant of UA159 (JAM1, deficient in manL) showed a more rapid resumption of growth following transfer from FMC-glucose to FMC-lactose, with a reduction in lag phase by as much as $3.5 \mathrm{~h}$. However, JAM1 transitioned from fructose to lactose with a lag time similar to UA159. Interestingly, the ElIMan mutant of GS-5 adapted much faster to growth on lactose from fructose ( $~ 6-h$ reduction in lag), but not from glucose to lactose, compared to the parental strain (Fig. 1A). These observations provide further evidence that metabolism of glucose or fructose can influence the imprinting of "memory" in the organisms that prevents the population from metabolizing lactose. It is interesting that strain-specific behaviors were observed in terms of the role of ElIMan in the behaviors of interest. We posit that growth on glucose results in the expression of genes that generate metabolic products that can promote lactose utilization by the bacterium, whereas the changes in gene expression and intermediates resulting from growth on fructose appear to significantly inhibit the expression or activity of gene products required for efficient transition to growth on lactose.

Experiments were also performed to exclude the possibility that cells that eventually grew in lactose after a prolonged lag were genetic variants, e.g., mutants that aberrantly regulated expression of lac genes. In particular, S. mutans GS-5 was cultivated overnight on FMC containing $20 \mathrm{mM}$ glucose or fructose, subcultured on FMC with 10 
TABLE 1 Growth characteristics of S. mutans wild-type strains, their isogenic mutants, and S. gordonii in FMC medium containing $10 \mathrm{mM}$ lactose $^{a}$

\begin{tabular}{|c|c|c|c|c|}
\hline \multirow[b]{2}{*}{ Strain/genotype } & \multicolumn{4}{|l|}{ Growth on lactose } \\
\hline & $\begin{array}{l}\text { Carbohydrate used } \\
\text { for preculture }\end{array}$ & $\begin{array}{l}\text { Avg } T_{d}(\min ) \\
\pm \mathrm{SD}\end{array}$ & $O D_{600}$ & $T_{\text {lag }}(\mathrm{h})$ \\
\hline \multicolumn{5}{|l|}{ S. mutans } \\
\hline \multirow[t]{5}{*}{ UA159 wild type } & Glucose & $66.0 \pm 2.1$ & 0.87 & 5.5 \\
\hline & Fructose & $74.9 \pm 5.7$ & 0.80 & 7.5 \\
\hline & Cellobiose & $96.1 \pm 6.7$ & 0.82 & 3.0 \\
\hline & Galactose & $56.2 \pm 5.1$ & 0.87 & 1.5 \\
\hline & Lactose & $52.7 \pm 4.4$ & 0.94 & 1.0 \\
\hline \multirow[t]{2}{*}{ JAM1 (manL) } & Glucose & $57.5 \pm 1.2$ & 0.81 & 2.0 \\
\hline & Fructose & $70.1 \pm 3.4$ & 0.91 & 7.0 \\
\hline \multirow[t]{2}{*}{$\operatorname{manL}$ celR } & Glucose & $66.3 \pm 3.9$ & 0.74 & 6.5 \\
\hline & Fructose & $69.0 \pm 2.6$ & 0.81 & 6.0 \\
\hline relA::Km & Glucose & $75.2 \pm 5.9$ & 0.74 & 10.5 \\
\hline$r e l A^{385}$ & Glucose & $80.6 \pm 3.6$ & 0.77 & 11.0 \\
\hline relp & Glucose & $63.2 \pm 3.0$ & 1.00 & 5.0 \\
\hline relQ & Glucose & $65.5 \pm 0.9$ & 0.98 & 5.5 \\
\hline $\operatorname{rel} A P Q$ & Glucose & $69.7 \pm 0.2$ & 0.99 & 8.0 \\
\hline \multirow[t]{5}{*}{ GS-5 wild type } & Glucose & $119.5 \pm 1.3$ & 0.84 & 1.5 \\
\hline & Fructose & $150.1 \pm 1.1$ & 0.73 & 11.5 \\
\hline & Cellobiose & $121.8 \pm 9.6$ & 0.70 & 2.5 \\
\hline & Galactose & $160.7 \pm 4.3$ & 0.58 & 2.0 \\
\hline & Lactose & $167.5 \pm 8.0$ & 0.66 & 2.0 \\
\hline \multirow[t]{2}{*}{ manL } & Glucose & $108.0 \pm 0.03$ & 0.83 & 2.0 \\
\hline & Fructose & $105.0 \pm 4.9$ & 0.84 & 5.5 \\
\hline \multirow[t]{2}{*}{ celR } & Glucose & $68.1 \pm 5.6$ & 0.90 & 6.0 \\
\hline & Fructose & $68.0 \pm 3.7$ & 0.93 & 6.0 \\
\hline \multirow[t]{2}{*}{ celA } & Glucose & $109.1 \pm 6.1$ & 1.13 & 2.5 \\
\hline & Fructose & $287.5 \pm 14.6$ & 0.48 & 9.5 \\
\hline \multirow[t]{2}{*}{$c e l B$} & Glucose & $183.5 \pm 14.7$ & 0.57 & 9.5 \\
\hline & Fructose & $180.2 \pm 1.0$ & 0.58 & 9.0 \\
\hline \multirow[t]{4}{*}{ S. gordonii DL1 } & Glucose & $49.6 \pm 3.0$ & 0.82 & 0.5 \\
\hline & Fructose & $51.8 \pm 3.4$ & 0.82 & 0.5 \\
\hline & Cellobiose & $48.4 \pm 3.5$ & 0.93 & 0.5 \\
\hline & Lactose & $50.1 \pm 4.0$ & 0.91 & 0.5 \\
\hline
\end{tabular}

${ }^{a}$ Each culture was started by 1:20 dilution into FMC-lactose of exponentially growing cultures prepared with FMC containing the specified carbohydrate. Each doubling time ( $T_{d}$ in min, average \pm standard deviation) was calculated as $T_{d}=\left(T_{2}-T_{1}\right) \times \log _{2} / \log \left(\mathrm{OD}_{2} / \mathrm{OD}_{1}\right)$ using data from the exponential phase $\left(\mathrm{OD}_{600}\right.$ of approximately 0.2 to 0.4$)$. The maximum optical density $\left(\mathrm{OD}_{600}\right)$ and the length of the lag phase $\left(T_{\text {lag }}\right)$ in hours reported are averages of results from at least three biological replicates. The end of the lag phase was defined as the point at which the $\mathrm{OD}_{600}$ reached 0.01 .

$\mathrm{mM}$ lactose to late exponential phase. Cultures were then spread on brain heart infusion (BHI) agar plates to form individual colonies, designated _G_L* and _F_L*, which were subsequently monitored for their growth behaviors during the transition from glucose or fructose to lactose. Consistent with the fact that these newly formed colonies were not genetic variants, the isolates behaved like the parental strain and (Fig. 1C) again showed that glucose-grown cells could transition onto lactose more rapidly than those cultured in fructose, as described above (Fig. 1A).

Memory effects from other carbohydrates. To better understand why glucose and fructose have different effects on lactose metabolism, we tested how other carbohydrates influence the transition to lactose, first by cultivating $S$. mutans strains to exponential phase in the sugars of interest and then by diluting the cultures into fresh FMC-lactose and monitoring growth. Cellobiose was tested out of the consideration that EllCel can function as an inducible glucose-PTS in S. mutans (7), and galactose was tested as it is primarily catabolized via the tagatose pathway, which is encoded in the lac operon and is the primary route for utilization of the galactose moiety of lactose. Lactose was also tested with the assumption that it should ensure optimal induction of the lac operon and that growth would initiate quickly after cells were transferred from FMC-lactose to the same medium. S. mutans UA159 cultivated in lactose or galactose 
rapidly transitioned onto lactose, with lactose-grown cells doing so significantly faster than glucose-grown cells and somewhat more rapidly than cells precultured in galactose (Table 1 and see Fig. S3A in the supplemental material). Interestingly, preculture on cellobiose also significantly enhanced the transition to lactose, more so than glucose, but not as much as lactose or galactose (Table 1).

Surprisingly, precultivation in cellobiose had the greatest impact on the ability of strain GS-5 to transition to lactose, with cultures showing a shorter lag and higher growth rate on lactose (Table 1 and see Fig. S3B in the supplemental material) than cells precultured on lactose or galactose. Again, precultivation with lactose or galactose had nearly identical effects, and the transition in this case was similar to cells that were first cultured in glucose. Therefore, similar to exposure to glucose, precultivation of $S$. mutans on cellobiose also significantly enhanced its capacity to transition to lactose compared to cells precultured with fructose. Notably, the PTS permeases for cellobiose (EllCel) and lactose (EIILac) share significant sequence similarities and in some organisms each permease can transport both cellobiose and lactose; as noted for Lactococcus lactis IL1403, where EIICel and a phospho- $\beta$-glucosidase (BgIS) cooperate to internalize then cleave lactose-6-P, respectively $(17,18)$. It is plausible, then, that the induction of expression of the cellobiose-PTS by glucose or cellobiose leads to enhanced growth on lactose because EIICel is already induced and can rapidly internalize lactose, thereby providing inducing intermediates that trigger lac gene expression.

Commensal oral streptococci do not display memory. As a constituent of the oral microbiota, S. mutans must compete against metabolically similar commensal bacteria for carbohydrates. To begin to investigate how memory in utilization of carbohydrates by $S$. mutans may influence oral microbial ecology, we performed the same experiments using a common oral commensal, Streptococcus gordonii DL1. Interestingly, DL1 grew efficiently on lactose when transitioning from glucose or fructose, with little difference in lag time, growth rate or final yield (Table 1 and see Fig. S4 in the supplemental material). Similar results were obtained when Streptococcus intermedius, Streptococcus sanguinis, or Streptococcus salivarius was evaluated under similar conditions (see Fig. S4 in the supplemental material), raising the question of whether the memory behavior may be unique to Streptococcus mutans and possibly to cariogenic streptococci. Interestingly, for another L. lactis strain, ATCC 11454, which is similar to IL1403, precultivation on glucose (compared to fructose) appeared to result in substantially improved growth on lactose (see Fig. S4 in the supplemental material). Of note, a recent study with Streptococcus pneumoniae demonstrated that the glucose-PTS encoded by $\operatorname{man} L M N$ is required for the catabolism of a number of secondary carbohydrates, potentially by serving as a multisubstrate transporter and facilitating the induction of necessary catabolic genes (19). However, a comparable role for ManLMN of $S$. mutans appears unlikely since mutations in the man operon result in better growth on nonpreferred carbohydrates.

Defects in cellobiose metabolism alter glucose-induced memory. In S. mutans, the expression of the cellobiose pathway, which includes two transcripts encoding celA and celBRCXD, is subject to EllMan-mediated repression when glucose is present. The operon is induced in the presence of cellobiose alone, or it can be induced with glucose in a strain lacking a functional EllMan. In the absence of ElIMan, EllCel becomes the primary glucose transporter (7). Induction of the cel operon is controlled by two phosphorylation events targeting the transcriptional activator, CelR. Phosphorylation of CelR by EllCel at His284 and His391 leads to inhibition of CelR activity, whereas phosphorylation by Enzyme I of the PTS at His226, His332, and His576 is required for CelR-dependent activation of the cel operon (7). When glucose is transported by the primary permease EIIMan, El preferentially phosphorylates EIIMan to optimize glucose utilization, which prevents induction of cel genes because CelR is not phosphorylated at His226, His332, and His576 by El. However, when EllCel is engaged in the transport of glucose, CelR is no longer phosphorylated at His284 and His391 by EllCel and is able to activate cel gene expression (7). 

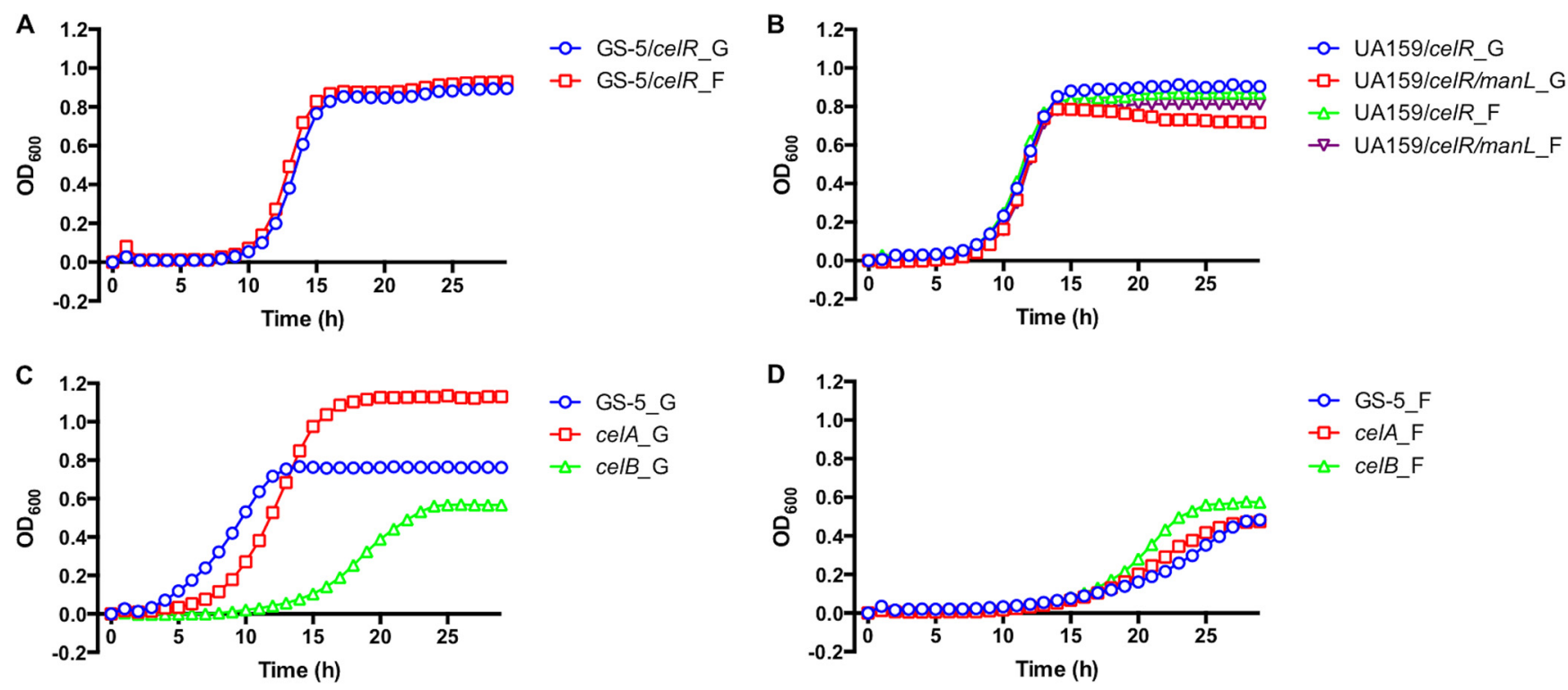

FIG 2 Disruption of the cellobiose pathway abolishes glucose memory. All strains were first cultivated in FMC containing 20 mM glucose (designated_G) or fructose $(F)$ to midexponential phase $\left(\mathrm{OD}_{600}=0.5\right)$ and then diluted 1:20 into FMC containing $10 \mathrm{mM}$ lactose for growth monitoring. (A) celR mutant of GS-5. (B) Mutants derived from UA159 that are deficient in celR or both celR and manL. (C and D) Wild-type strain GS-5 and its otherwise-isogenic mutants deficient in phospho- $\beta$-glucosidase (celA) or EllB of the cellobiose-PTS (celB) were grown in FMC with glucose (C) or fructose (D) before inoculation.

We first investigated the effects of a celR deletion on memory. As shown in Fig. 2A, a celR mutant of GS-5 no longer displayed enhanced growth on lactose when transitioning from glucose, and the mutant retained the slow transition from fructose to lactose noted with the parental strain. The same was true when a celR deletion was introduced into an EIIMan mutant of UA159 (UA159/manL/celR, Fig. 2B). However, the celR mutant of GS-5 (but not that of UA159) displayed a significant reduction in doubling time during exponential phase in comparison to the wild type (Table 1). Collectively, these results affirmed the notion that the glucose-associated impact on the transition to lactose occurs through the cellobiose pathway. To further differentiate the contribution to lactose metabolism by the EIICel (celBCD) and the phospho- $\beta$ glucosidase (celA), mutants deficient in celB or celA were constructed in strain GS-5. Growth tests using these mutants indicated that loss of celA resulted in slightly longer transition to, but significant enhancement in yield on, lactose (Fig. $2 \mathrm{C}$ and D). Loss of EllCel (celB) drastically increased the length of the lag (by $\sim 8 \mathrm{~h}$ ) required for GS-5 to transition from glucose onto lactose (Fig. $2 \mathrm{C}$ and D), suggesting that EllCel plays a more prominent role than does phospho- $\beta$-glucosidase (CelA) in lactose metabolism.

When fully induced, the cellobiose pathway of $L$. lactis alone is sufficient for growth on medium containing lactose as the carbohydrate source (18). It was posited that lactose is internalized by the cellobiose-PTS and phosphorylated on the glucose moiety, instead of on the galactose moiety, the latter being the case for lactose internalized by the lactose-PTS. Subsequent hydrolysis of the phosphorylated lactose moiety by BglS yields glucose-6-phosphate (G-6-P) and galactose, with galactose being catabolized by the Leloir pathway. To determine whether a similar pathway exists in S. mutans, mutants deficient in lactose catabolic enzymes were constructed in the UA159 and GS-5 genetic backgrounds. Interestingly, the isogenic mutants of UA159 defective in EllLac (IacE) or the phospho- $\beta$-galactosidase (IacG) failed to grow on lactose, even when precultured with cellobiose (see Fig. S5 in the supplemental material). The same was true for the mutants derived from GS-5. Nevertheless, a previous analysis of lactose and galactose metabolism by S. mutans UA159 indicated a substantial induction ( $\sim$-fold increase in mRNA levels) of the Leloir pathway genes in cells growing on lactose compared to glucose, and such induction generally requires that the cells internalize galactose (9). Taken together, these findings suggest that the cellobiose pathway in $S$. 
A

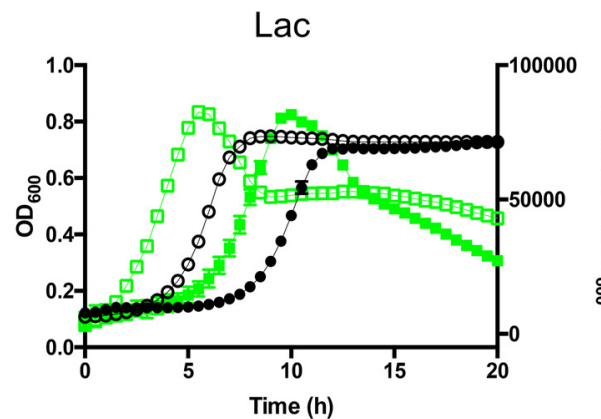

-- OD_UA159 -- GFP_UA159

B

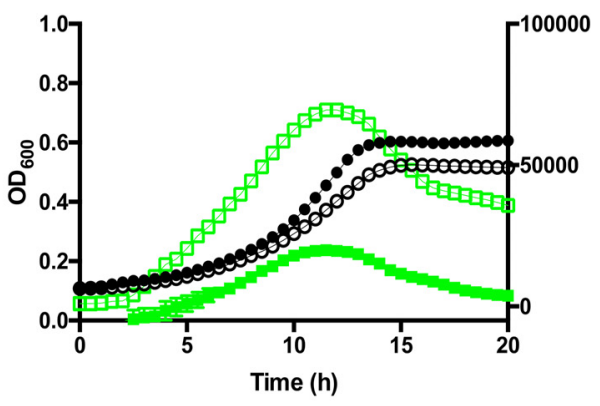

- OD_GS-5 - GFP_GS-5
○ OD_manLMN - GFP_manLMN

Lac

○ OD_manL - GFP_manL

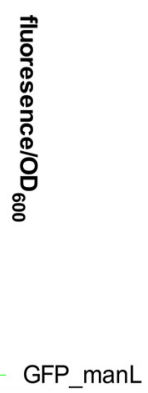

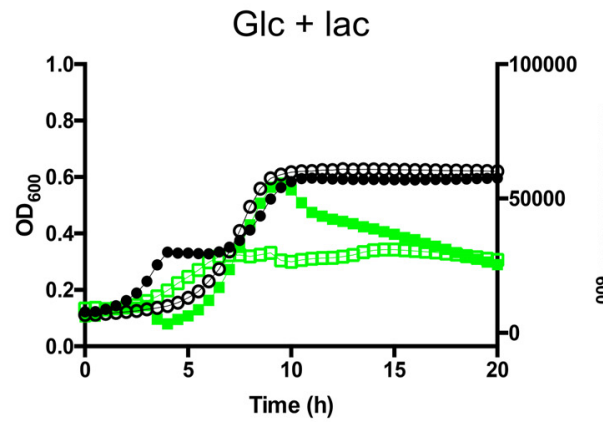

๑- OD_manLMN

FIG 3 Growth curves $\left(\mathrm{OD}_{600}\right.$, black) and expression of PlacA::gfp fusion (relative fluorescence, green) by strains in the UA159 (A) and GS-5 (B) backgrounds. Wild-type strains $(O)$ and mutants deficient in EllMan $(O)$ were cultivated to exponential phase in FMC containing 20 mM glucose and then diluted into FMC

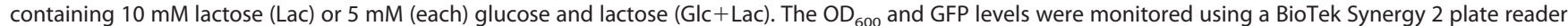
maintained at $37^{\circ} \mathrm{C}$. See Materials and Methods for information on normalization of the data.

mutans is not an independent, secondary catabolic pathway for lactose, but that the cellobiose PTS has a significant role in regulation of the catabolism of lactose, apparently by transporting and concomitantly phosphorylating lactose.

Strain-specific effects of ElIMan in regulating lac gene expression. Growth phenotypes of the Ell Man mutants so far have shown alterations in the transition onto lactose under certain conditions (Fig. 1), which could be caused by relaxed repression of the lac operon, and in particular of the genes encoding the enzymes of the tagatose pathway $(\operatorname{Iac} A B C D)$. To test this theory, expression of the lac operon was investigated using a PlacA::gfp promoter fusion established in the wild-type and EllMan-deficient genetic backgrounds. Of note, we identified that there was a potentially significant difference in the sequence of the regions upstream of the lacA gene in strains UA159 and GS-5. In particular, two contiguous $(\mathrm{T})_{7} \mathrm{G}$ motifs are present 193 nucleotides upstream of lacA in GS-5, but only one is found in UA159 (see Fig. S6 in the supplemental material), and no divergence is found in the rest of the lacA sequence. We therefore created two separate versions of $g f p$-promoter fusions, one containing two $(\mathrm{T})_{7} \mathrm{G}$ motifs and the other containing one, and assessed green fluorescent protein (GFP) expression from both constructs in their cognate genetic backgrounds. Strains were first cultivated to mid-exponential phase in FMC-glucose and then diluted into FMC containing only lactose or a mixture of equal concentrations of lactose and glucose. Growth and GFP levels were then monitored in real-time during in a BioTek Synergy plate reader (Fig. 3).

Compared to what was seen in the wild-type UA159 genetic background, the EIIMan mutant ( $\operatorname{man} L M N$; and similarly $m a n L$, data not shown) showed a more rapid induction of the lac operon (by $\sim 5 \mathrm{~h}$ ), although the maximal levels of expression of GFP measured were similar in both strains (Fig. 3A). When the cells were monitored in a medium containing $5 \mathrm{mM}$ glucose and $5 \mathrm{mM}$ lactose, the wild type expectedly showed 


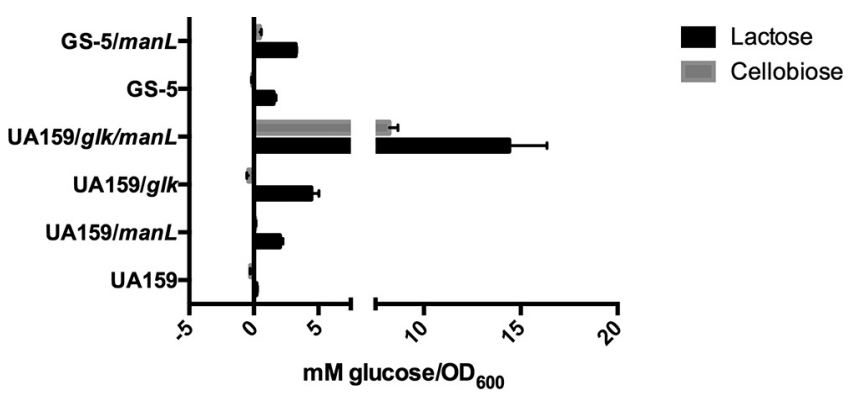

FIG 4 Detection of free glucose in the cultures of $S$. mutans growing on lactose or cellobiose. Wild-type strains UA159 and GS-5, as well as their isogenic mutants deficient in glucose-PTS ( $m a n L$ ) or glucokinase ( $g / k$ ) or both, were cultured in FMC containing $10 \mathrm{mM}$ lactose or cellobiose to midexponential phase, and then supernates of the cultures were obtained after centrifugation and filtration. The levels of glucose in these supernates were measured using glucose assay kits. Each bar represents the average of three independent experiments that were each repeated at least twice, with error bars denoting the standard deviations.

delayed lac expression until the onset of diauxie. In contrast, the Ellman mutants presented no diauxie and showed constitutive, albeit significantly lower expression of the PlacA::gfp fusion, which started to increase early during growth but quickly plateaued in the mid-exponential phase of growth $\left(\mathrm{OD}\right.$ at $\left.600 \mathrm{~nm}\left[\mathrm{OD}_{600}\right] \approx 0.3\right)$, reaching levels significantly lower than in the wild-type background (Fig. $3 \mathrm{~A}$ ). While the early induction of the lac operon in the Ell ${ }^{\text {Mn }}$-negative background can be viewed as a result of loss of inducer exclusion, the premature halt of such induction cannot be attributed simply to a loss of CCR, since the impact would have been the opposite.

Different from UA159, a GS-5 variant carrying its cognate PlacA::gfp promoter fusion, the one with two $(T)_{7} G$ motifs, produced generally lower GFP signals under the same conditions in comparison with UA159 carrying its cognate PlacA::gfp fusion. Importantly, the EIIMan mutant (manL) of GS-5 produced markedly higher expression of PlacA::gfp fusion, compared to the wild type (Fig. 3B), while growing on lactose alone or in medium containing both lactose and glucose. However, as indicated earlier (Fig. $1 \mathrm{~A})$, this mutant displayed neither a shorter lag nor earlier lac gene induction during the transition from glucose to lactose. This phenotype will be discussed further below.

Release of free glucose during lactose metabolism and its impact on gene regulation. In S. mutans, lactose, cellobiose, and maltose are internalized by the PTS, and the resultant phosphodisaccharides are cleaved to generate phosphorylated hexose and free glucose $(7,9,20)$. The glucose thus produced can enter glycolysis following phosphorylation by a glucokinase ( $g / k)$. Recent studies of the metabolism of maltose and maltooligosaccharides by $S$. mutans suggest that significant amounts of glucose are released into the environment and reinternalized by the PTS (21). We reported a similar release of free hexoses (fructose) when sucrose is catabolized through the sucrose-specific PTS by $S$. mutans $(12,13)$. Here, we show that when cultivated on lactose alone, free glucose can be detected in the supernates of the manL mutants in concentrations ranging from 0.5 to $1.5 \mathrm{mM} / \mathrm{OD}_{600}$. However, high levels of free glucose were not detectable in the supernatant fluid of the parental strain UA159, presumably because of reinternalization of the glucose by EllMan in the wild type (Fig. 4). Notably, the concentrations of glucose that accumulated in the supernates were higher in strains carrying a deletion of $g / k$, both in the wild-type and in the manL mutant genetic backgrounds (Fig. 4). Importantly, the presence of free glucose in the extracellular environment could affect the transcription of the cellobiose and lac operons. Specifically for UA159, small amounts of glucose transported by EllCel could induce a basal level expression of the cellobiose pathway without triggering CCR, and the cel gene products could then internalize lactose, similar to what is observed in $L$. lactis, but without generating Gal-6-P to induce the lac operon. In the absence of an intact ElIMan, a situation that forces glucose internalization through EllCel, extracellular glucose would further enhance the activities of the cellobiose pathway, significantly 


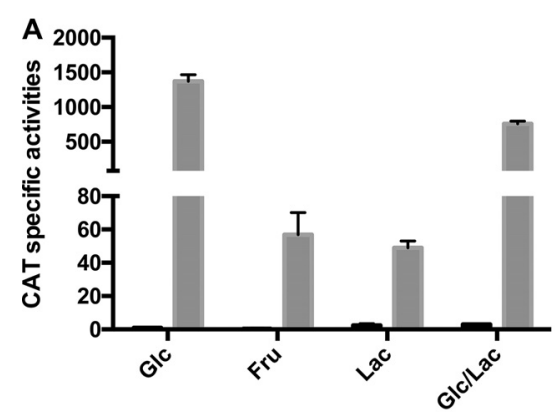

Sugar in growth media

B

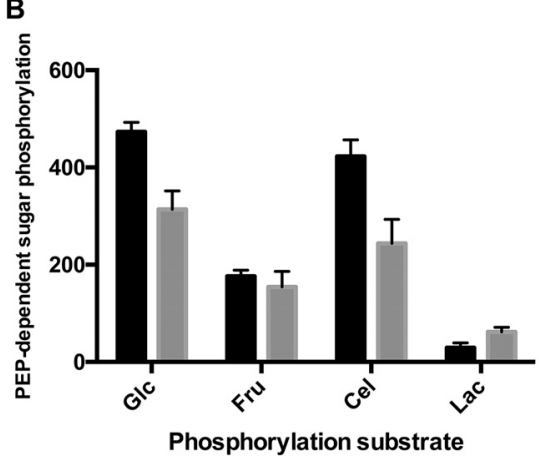

UA159_G

manL_G

FIG 5 Expression of the cellobiose pathway by cells growing in the presence of lactose. (A) The activities of a PcelA::cat reporter fusion were measured in wild-type UA159 or its manL mutant, with cells growing on glucose (Glc), fructose (Fru), lactose (Lac), or a mixture of $5 \mathrm{mM}$ (each) glucose and lactose. (B) PTS-dependent sugar phosphorylation assays were performed to measure the PTS activities transporting glucose, fructose, cellobiose (Cel), and lactose. Wild-type strains GS-5 and UA159 were cultured in FMC containing a mixture of $5 \mathrm{mM}$ (each) glucose and lactose before assay. All bars represent the averages from three independent experiments with each measurement repeated twice. The error bars represent the standard deviations.

diverting the influx of lactose through the Cel system and resulting in downregulation of the lac operon. This hypothesis was supported by the reduced expression of the PlacA::gfp fusion in the EllMan mutant compared to cells with the wild-type genetic background, when growing on a mixture of both glucose and lactose (Fig. 3A).

To study the activities of the cellobiose operon, a PcelA::cat promoter fusion was tested in the manL mutant. In agreement with our hypothesis, the PcelA::cat fusion in the manL mutant of UA159 presented significantly increased activity compared to the wild type when growing on lactose, but especially when growing on a mixture of glucose and lactose (Fig. 5A). Furthermore, we have observed an apparent preference by both UA159 and GS-5, when growing on a mixture of equal amounts of glucose and lactose, to express the glucose and cellobiose PTS operons, rather than the lactose-PTS (Fig. 5B).

In contrast, significantly more glucose was released by GS-5 than UA159 when growing on lactose $\left(>1 \mathrm{mM} / \mathrm{OD}_{600}\right)$ even for the wild type (Fig. 4). A manL mutant in the GS-5 genetic background accumulated more extracellular glucose $\left(>3 \mathrm{mM} / \mathrm{OD}_{600}\right)$ than the wild type. As reinternalization of glucose via EllMan creates glucose-6phosphate and other catabolites (e.g., F-1,6-bP) capable of triggering the activity of CcpA (2), the relaxed expression of the lac genes by the manL mutant of GS-5 is consistent with the conventional model for CCR. Therefore, it is plausible that the amount of glucose released during lactose metabolism is sufficient to trigger CcpAdependent CCR in GS-5, as we observed in a previous study (6).

In light of the sequence divergence between the lacA promoters in GS-5 and UA159, the PlacA::gfp fusion created using the UA159 lacA promoter template, namely, the version harboring only one $(T)_{7} G$ motif (see Fig. $S 6$ in the supplemental material), was introduced into the backgrounds of GS-5 and its manL mutant (Fig. 6). Interestingly, the 


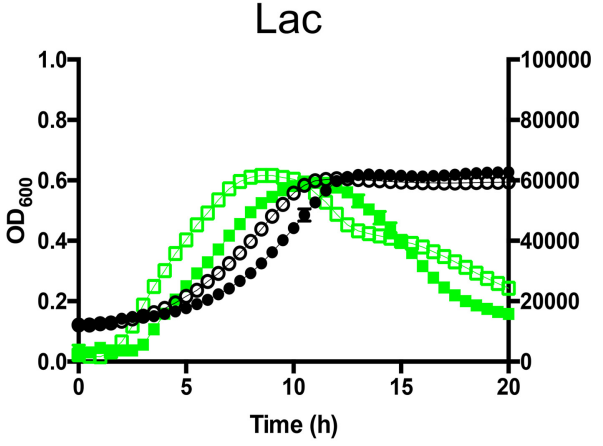

- OD_GS-5* - ${ }^{*}$ GFP_GS-5*
๑ OD_manL* $\square$ GFP_manL*

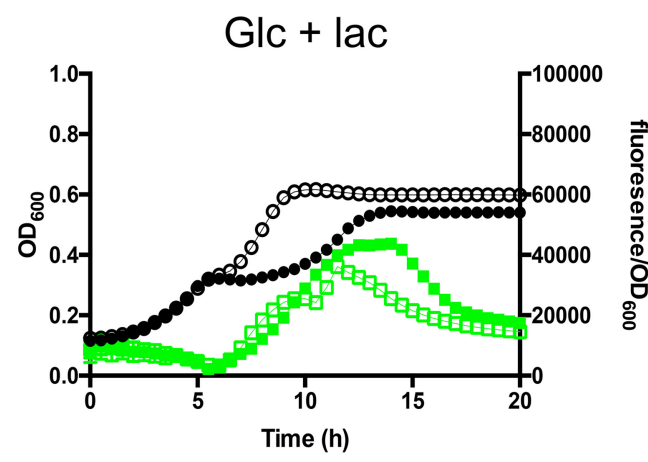

- OD_GS-5* - ${ }^{*}$ GFP_GS-5*
○ OD_manL* $\square$ GFP_manL*

FIG 6 Expression of a UA159-derived PlacA::gfp fusion (relative fluorescence, green) in the GS-5 background. Wild type $(\bullet)$ and a manL mutant $(O)$ were cultivated to exponential phase in FMC containing $20 \mathrm{mM}$ glucose and then diluted into FMC containing either $10 \mathrm{mM}$ lactose (Lac) or $5 \mathrm{mM}$ (each) glucose and lactose (Glc+Lac). $\mathrm{OD}_{600}$ and GFP fluorescence were monitored using a BioTek Synergy 2 plate reader maintained at $37^{\circ} \mathrm{C}$.

manL mutant no longer showed enhanced expression of the reporter fusion when growing in the presence of lactose; instead it produced lower GFP signals than the wild type when cultured in a combination of glucose and lactose. In other words, the lacA expression patterns in these two strains now resembled those in the UA159 background. These results indicate that the difference in lactose metabolism observed between UA159 and GS-5 is partly attributable to how each lac operon is regulated by the glucose PTS. However, a brief analysis of the lacA promoter region in both strains failed to locate a conserved CcpA-binding site, but the potential that the $(T)_{7} G$ motif acts as cis-acting regulatory elements is supported by the data.

Lastly, when cellobiose or trehalose was used as the sole carbohydrate source to cultivate S. mutans deficient in EllMan, free glucose was again detected in the supernates, albeit at levels lower than what was released by lactose-grown cultures (Fig. 4 and data not shown). The cause for this difference has not been determined but could reflect that glucokinase activity is increased when cells are cultured on disaccharides composed only of glucose.

Free glucose and other preferred carbohydrates are required for efficient transition to growth on lactose. When exponential-phase cultures of $S$. mutans growing in FMC with $20 \mathrm{mM}$ glucose or fructose were diluted 1:20 into fresh FMC containing lactose, carryover of residual glucose or fructose occurs, which could influence growth behaviors. To eliminate carryover of sugars and assess the impact of small amounts of hexose on the transition to lactose, cells were harvested in exponential phase and washed extensively with sterile phosphate-buffered saline (PBS), then small amounts $(0,0.125$, and $0.25 \mathrm{mM})$ of glucose or other monosaccharides were added into the FMC-lactose medium before inoculation. Unexpectedly, without addition of the monosaccharides, washed cultures of $S$. mutans displayed an apparent growth arrest that lasted as long as $24 \mathrm{~h}$ (extended memory [Fig. 7; see also Fig. $\mathrm{S7}$ in the supplemental material for more $S$. mutans isolates and commensals]). Such growth arrest was particularly significant when the precultures were prepared using FMCfructose. However, addition of small amounts of the glucose, fructose, mannose, glucosamine (GlcN), or $\mathrm{N}$-acetylglucosamine (GlcNAc) accelerated the transition of $S$. mutans to growth on lactose, drastically shortening the lag phase (Fig. 7). The effects of sugars on the duration of the lag phase were concentration dependent and could be observed at levels of hexose as low as $10 \mu \mathrm{M}$ (see Fig. $\mathrm{S} 8$ in the supplemental material). Importantly, mutants lacking the PTS permease(s) for the cognate monosaccharides showed reduced or abolished responses to low levels of these hexoses (data not shown). When other disaccharides besides lactose were tested in this experiment, it was determined that similar effects on the lag phase for transition to growth on lactose could be observed for cellobiose or trehalose, but not for sucrose or maltose (data not shown). Certain S. mutans strains, however, were able to grow efficiently on some of 


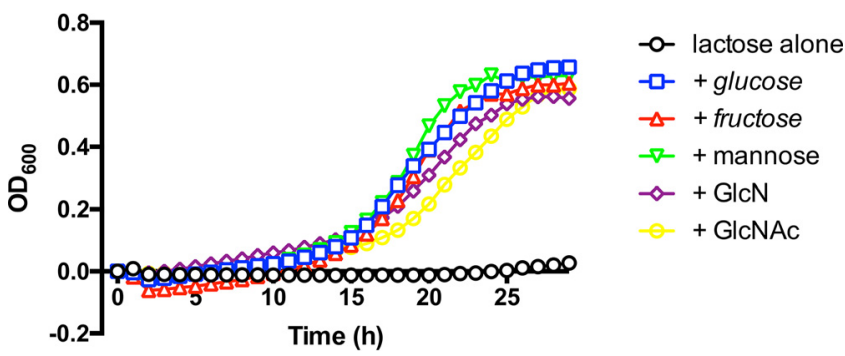

FIG 7 S. mutans requires preferred monosaccharides for growth on lactose. GS-5 was cultured to exponential phase in FMC that contained $20 \mathrm{mM}$ glucose, washed three times with sterile PBS, resuspended in the same buffer, and then diluted at a 1:20 ratio into fresh FMC containing either $10 \mathrm{mM}$ lactose or $10 \mathrm{mM}$ lactose in addition to $0.125 \mathrm{mM}$ glucose, fructose, mannose, GIcN, or GlcNAc for growth monitoring in a Bioscreen C.

these disaccharides alone, e.g., GS-5 on cellobiose. The fact that carbohydrates other than glucose were able to rescue growth on lactose suggests a more general effect by these preferred carbohydrates that impacts lactose metabolism, one that is likely independent of the cellobiose pathway discussed above.

Also of particular interest was the fact that the addition of galactose (as much as $0.25 \mathrm{mM}$ ) to any of the washed $S$. mutans cultures failed to rescue growth on lactose, even though a number of these isolates harbored genes encoding a high-affinity galactose-specific PTS permease (data not shown) (22). Further experimentation revealed that these isolates also showed a similar growth arrest when washed prior to transferring from glucose to galactose as the sole carbohydrate, a defect that again could be rescued by the addition of small amounts of glucose (data not shown).

Collectively, these results indicate the existence of distinct hierarchies in the preference for utilization of common carbohydrates by S. mutans, with most monosaccharides, sucrose and maltose being preferred, followed by trehalose, cellobiose, galactose, and lactose. Since $S$. mutans cultures have been shown to release and reinternalize glucose when growing on lactose, cellobiose, or trehalose, often at concentrations that were comparable to what were used to elicit repression of genes for nonpreferred carbohydrates, these findings suggest concentration-dependent and disparate effects of glucose and the glucose-PTS on catabolism of these less-preferred carbohydrates: the expression of the catabolic genes is negatively regulated by the glucose porter via CCR at higher concentrations of glucose, but efficient induction is dependent on small amounts of glucose, and apparently certain other sugars, for activation. The molecular mechanisms responsible for such activation remains to be elucidated, although it is worth noting that a positive-feedback loop generally exists for the induction of these pathways $(7,9)$, where the signal output of these self-activating circuits could be enhanced not only by the substrates (for EllCel) or metabolic intermediates (for EllLac and EII Tre) but also by triggering efficient expression of the PTS and the glycohydrolase required for catabolism of the nonpreferred carbohydrate.

rel mutants showed an altered ability to transition onto lactose. When faced with environmental stresses, such as amino acid starvation, bacteria often respond by synthesizing tetra- and pentaphosphorylated guanosine alarmones, collectively designated (p)ppGpp, that can suppress protein biosynthesis and alter gene regulation (23). There are three (p)ppGpp-metabolizing enzymes in S. mutans: RelA, which has both synthase and hydrolase activities, and two synthase-only enzymes, RelP and RelQ, which contribute to (p)ppGpp pools under specific circumstances (24). To examine the possibility that the stringent response is involved in the transition of $S$. mutans from one carbohydrate source to another, we studied the growth behaviors of a set of otherwiseisogenic mutants that lacked individual or combinations of the rel genes. As shown in Fig. 8 and Table 1, deletion of relP or relQ had no apparent impact on the ability of UA159 to transition from glucose onto lactose. On the other hand, deletion of the entirety of relA via allelic exchange $(r e / A:: K m)$ or the disruption of the (p)ppGpp- 


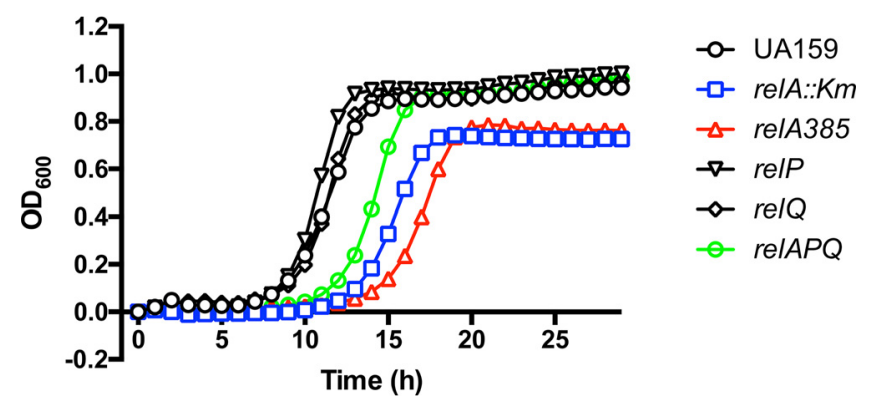

FIG 8 Mutations in (p)ppGpp-metabolizing enzymes affect growth of S. mutans on lactose after transferring from glucose. Wild-type strain UA159 and its isogenic mutants with complete deletion of relA (relA::Km), relP, relQ, all three (relAPQ), or a truncation in relA (relA ${ }^{385}$ [relA385]) that abolishes its (p)ppGpp-hydrolase activities were each cultured in FMC containing $20 \mathrm{mM}$ glucose to midexponential phase and then diluted 1:20 into fresh FMC constituted with $10 \mathrm{mM}$ lactose for growth monitoring.

hydrolase function alone (with a truncation beginning at position 385 [rel $\left.A^{385}\right]$ ) (25) resulted in an increase in the transition time for growth on lactose. Further, a strain missing all three rel enzymes ( $\triangle$ relAPQ), which appears to produce no (p)ppGpp (24), also showed a similar increase in the time to transition to growth on lactose. Similar results were obtained from these mutants when the precultures were prepared using FMC-fructose (data not shown). Previous work from our group characterizing the enzymatic activities of these mutants has indicated that while the triple-null mutant produces no (p)ppGpp, loss of the hydrolase activity of RelA ( $\left.r e l A^{385}\right)$ or deletion of relA entirely results in higher basal levels of alarmones (25). Therefore, it appears that modest changes in (p)ppGpp pools resulting from loss of, or changes in the synthase/ hydrolase capabilities of RelA, could significantly affect the ability of S. mutans to transition from a preferred carbohydrate onto a nonpreferred source. It should be noted that our group has demonstrated that growth on fructose, compared to glucose, induces changes in the transcriptome that alter the expression of many stress-related genes (13) and that accumulation of fructose-phosphate (14) and other sugar phosphates (26) could lead to growth arrest by S. mutans. Of note, Escherichia coli has a requirement for an intact stringent response to cope with sugar-phosphate stress (27). We hypothesize that the PTS and presence of certain carbohydrates can contribute to prolonged growth arrest by altering ( $p$ )ppGpp pools and that the effects can be influenced by the activity of PTS enzymes involved in the transport of preferred carbohydrates.

Summary and relevance to oral microbial ecology. Biofilms in the human oral cavity experience frequent and substantial shifts in the availability and type of carbohydrates, and most bacteria depend on CCR to prioritize sugar metabolism. CCR in S. mutans relies heavily on the PTS and micromolar levels of preferred carbohydrates can trigger PTS-dependent CCR for genes for secondary metabolites, such as fruA encoding a fructan hydrolase (6). Here, we investigate the unusual phenomenon of long-term memory and explored the molecular basis for the delay in adaptation by S. mutans to the introduction of lactose depending upon prior exposure to glucose or fructose. We also show that $S$. mutans requires low levels of preferred carbohydrates (including glucose) for efficient induction of the lac operon, notable also because substantial amounts of free glucose are released into the environment by cells that are actively catabolizing lactose (as well as cellobiose and trehalose). Therefore, as a by-product of metabolism of lactose and other disaccharides, free glucose at low concentrations may serve as a signal to prime the induction of the lac operon; however, higher levels of glucose or other preferred carbohydrates could downregulate lac expression through CCR. This phenomenon echoes a recent report wherein the glucose-PTS of Streptococcus pneumoniae was shown to serve as a surveyor of external carbohydrates that is required for the metabolism of multiple carbohydrates (19). Unlike for the pneumococcus, fructose, which is frequently present in human diets but is also a by-product of 
sucrose metabolism by extracellular enzymes and the PTS of S. mutans (12), could be viewed as a negative signal for the induction of the lac system. We posit that, as a bet-hedging strategy, certain populations of S. mutans respond to a combination of these hexoses in a way that delays the expression of the lac operon in anticipation of future ingestion of fructose or sucrose by the host, in order to avoid the energy expenditure required for synthesizing enzymes for intermittently available, lesspreferred carbohydrates. We have previously shown that growth on fructose or sucrose downregulates the expression of enzymes required for glycogen metabolism (SMU.1535 to SMU.1539) (13), whereas a FruK (1-phosphofructokinase)-deficient mutant overexpresses some of the same enzymes (SMU.1536 to SMU.1539), presumably in response to increased levels of fructose-1-phosphate (14). It remains to be clarified how these changes in glycogen-related gene expression affect the persistence of the bacterium under these conditions. Finally, this study raises the question of how free hexoses released during metabolism of these disaccharides influence oral biofilm ecology, since other microorganisms in the same niche can be provided with preferred carbohydrates when $S$. mutans and possibly other oral streptococci metabolize disaccharides. One scenario of particular interest to us is the emergence of so-called cheaters within the population of $S$. mutans that take advantage of these free hexoses without investing in the production of the enzymes necessary for catabolizing lactose or other disaccharides, as indicated by our preliminary study (Fig. 5B and data not shown). Further research at the single-cell level could help to shed more light on the behavior of individual cells under these conditions.

\section{MATERIALS AND METHODS}

Bacterial strains, media, and culture conditions. Streptococcus mutans, including wild-type and mutant strains, plus five other strains of lactic acid bacteria (LAB) (Table 2), were maintained on BHI (Difco Laboratories, Detroit, $\mathrm{MI}$ ) agar plates or in liquid medium at $37^{\circ} \mathrm{C}$ in a $5 \% \mathrm{CO}_{2}$, aerobic atmosphere. Antibiotics were added to the media, when necessary, as follows: kanamycin $\left(0.5\right.$ to $\left.1 \mathrm{mg} \mathrm{ml}^{-1}\right)$, erythromycin (5 to $\left.10 \mu \mathrm{g} \mathrm{ml}^{-1}\right)$, spectinomycin $\left(0.5\right.$ to $\left.1 \mathrm{mg} \mathrm{ml}^{-1}\right)$, and tetracycline $\left(10 \mu \mathrm{g} \mathrm{ml}^{-1}\right)$. E. coli $\mathrm{DH} 10 \mathrm{~B}$ was used as a cloning host and cultivated in $\mathrm{L}$ broth at $37^{\circ} \mathrm{C}$ with agitation in air. When needed, $\mathrm{L}$ broth was supplemented with the antibiotics kanamycin $\left(50 \mu \mathrm{g} \mathrm{ml}^{-1}\right)$ and erythromycin (200 $\left.\mu \mathrm{g} \mathrm{ml}^{-1}\right)$. For most experiments, S. mutans and other LAB were cultured in a modified version of the chemically defined medium FMC (28) formulated with various carbohydrates (Thermo Fisher Scientific, Waltham, MA; MilliporeSigma, St. Louis, MO), as indicated.

To monitor bacterial growth, all strains were cultivated overnight in $5 \% \mathrm{CO}_{2}$ in $\mathrm{FMC}$ containing the specified carbohydrate, diluted 1:10 into the same medium, and cultured until the $\mathrm{OD}_{600}$ reached 0.5 . Then, the cultures were again diluted (1:20) into fresh FMC constituted with the desired carbohydrates and incubated at $37^{\circ} \mathrm{C}$ in a Bioscreen C Lab system (Helsinki, Finland). Each experiment included three individual isolates (biological replicates) in triplicate (technical replicates); each sample $(300 \mu \mathrm{l})$ was overlaid with sterile mineral oil $(60 \mu \mathrm{l})$ to reduce exposure to air, and the $\mathrm{OD}_{600}$ was measured every hour after a brief agitation. In experiments designed to eliminate carryover of sugar(s) from the prior incubation when cultures were diluted, exponential-phase cultures were first cooled on ice, harvested by centrifugation and then washed three times with an equal volume of cold, sterile PBS. The cultures were then resuspended in the desired medium, diluted as necessary, and growth was monitored as described above.

For bacteria containing a PlacA::gfp fusion, cells were cultured as described above to exponential phase and diluted 1:20 into $200 \mu \mathrm{l}$ of FMC medium in a Costar (Corning, Corning, NY) 96-well microtiter plate with a clear bottom, and the culture was overlaid with $60 \mu \mathrm{l}$ of mineral oil. The growth of the bacteria $\left(\mathrm{OD}_{600}\right)$ and expression of the promoter fusion as represented by green fluorescent light emission (excitation, 485/20 nm; emission, 528/20 nm) were monitored simultaneously every 30 min for $20 \mathrm{~h}$ using a fluorescence-capable Synergy 2 Multi-Mode reader from BioTek (Winooski, VT). For each test strain, a control strain for background subtraction was included; the control had the same genetic background but lacked the reporter fusion. To calculate the relative expression of the promoter fusion, the background fluorescence of the control sample was subtracted from each raw reading of the test sample and then divided by the corresponding $\mathrm{OD}_{600}$ value for normalization. Finally, cultures used to measure the glucose in culture supernates and for chloramphenicol acetyltransferase (CAT) and PTS assays were also prepared by diluting from an overnight culture using fresh FMC containing the specified carbohydrate(s), followed by incubation until the $\mathrm{OD}_{600}$ reached 0.5 .

Construction of genetic mutants and promoter::gfp fusions. Standard recombinant DNA techniques were employed to engineer plasmids and mutant strains. All restriction enzymes were purchased from New England BioLabs (Beverly, MA) and used as directed by the supplier. DNA preparation was performed using QIAquick DNA purification kits supplied by Qiagen (Valencia, CA). All DNA oligonucleotides were synthesized by Integrated DNA Technologies (Coralville, IA) and are listed in Table 3. 
TABLE 2 Bacterial strains used in this study

\begin{tabular}{|c|c|c|}
\hline Strain & Relevant characteristics ${ }^{a}$ & Source or reference \\
\hline \multicolumn{3}{|l|}{ Streptococcus mutans } \\
\hline UA159 & Wild type, serotype $c$ & University of Alabama \\
\hline JAM1 & UA159 manL::Km & 35 \\
\hline MMZ540 & UA159 celR::Spr manL::Emr & 7 \\
\hline JLrelA & UA159 relA::Km & 36 \\
\hline relA $A^{385}$ & $\begin{array}{l}\text { UA159 containing a truncation of } \\
\text { relA starting at residue } 385\end{array}$ & 25 \\
\hline JLrelP & UA159 relP::Km & 24 \\
\hline JLrelQ & UA159 relQ::Kmr & 24 \\
\hline JLT1 ( $\triangle$ relAPQ) & UA159 relA::Em relP::Spr relQ::Km & 24 \\
\hline MMZ712 & UA159 lacE::Em & 9 \\
\hline MMZ702 & UA159 lacG::Emr & 9 \\
\hline MMZ1285 & UA159 PlacA::gfp & This study \\
\hline MMZ1287 & UA159 manLMN::Emr PlacA::gfp & This study \\
\hline MMZ1548 & UA159 manL::Emr PlacA::gfp & This study \\
\hline MMZ493 & UA159 PcelA::cat & 7 \\
\hline MMZ495 & UA159 manL::Em ${ }^{r}$ PcelA::cat & 7 \\
\hline MMZ1422 & UA159 glk::Kmr & This study \\
\hline MMZ1455 & UA159 glk::Km manL::Em ${ }^{r}$ & This study \\
\hline GS-5 & Wild type, serotype $c$ & R. J. Gibbons \\
\hline MMZ1462 & GS-5 manL:::Em ${ }^{r}$ & This study \\
\hline MMZ1424 & GS-5 celR::Kmr & This study \\
\hline MMZ1509 & GS-5 celA::Em ${ }^{r}$ & This study \\
\hline MMZ1504 & GS-5 celB::Emr & This study \\
\hline MMZ1510 & GS-5 lacE::Em & This study \\
\hline MMZ1517 & GS-5 lacG::Emr & This study \\
\hline MMZ1480 & GS-5 PlacA::gfp & This study \\
\hline MMZ1483 & GS-5 manL::Em PlacA::gfp & This study \\
\hline LM-7 & Wild type, serotype e & A. Eisenberg \\
\hline V403 & Wild type, serotype c & T. Kitten \\
\hline 10449 & Wild type, serotype c & A. Eisenberg \\
\hline Smu44 (11VS1) & Wild type, serotype e & 37 \\
\hline Smu33 (11SSST2) & Wild type, serotype c, EllGal+ & 37 \\
\hline Smu21 (1SM1) & Wild type, serotype e, EllGal+ & 37 \\
\hline UA101 & Wild type, serotype c & Laboratory strain \\
\hline OMZ175 & Wild type, serotype f, EllGal + & B. Guggenheim \\
\hline \multicolumn{3}{|l|}{ Other strains } \\
\hline Streptococcus gordonii DL1 & Wild-type standard strain & ATCC 49818 \\
\hline Streptococcus sanguinis SK150 & Wild-type standard strain & Laboratory stock \\
\hline Streptococcus intermedius & Wild-type standard strain & ATCC 31412 \\
\hline Streptococcus salivarius 57.I & Wild-type standard strain & 38 \\
\hline Lactococcus lactis & Wild-type standard strain & ATCC 11454 \\
\hline
\end{tabular}

${ }^{a} \mathrm{Km}^{\mathrm{r}}$, kanamycin resistance; $\mathrm{Sp}^{r}$, spectinomycin resistance; $\mathrm{Em}^{\mathrm{r}}$, erythromycin resistance; EllGal + , containing EllGal.

The majority of the mutant derivatives of $S$. mutans used in this study were engineered by following the PCR-ligation approach (29) utilizing three genetic cassettes encoding resistance to kanamycin (Km), erythromycin (Em), or spectinomycin (Sp). To construct allelic exchange mutants of lacG and $g / k$, however, two PCR products flanking each target gene were created so that sufficient sequence overlap ( $>25 \mathrm{bp}$ ) existed between these DNA fragments and the antibiotic cassette (see Table 3 for details). A Gibson Assembly Mastermix (New England BioLabs) was then utilized to ligate these DNA fragments in a one-step, 1-h reaction before transformation of $S$. mutans. Based on the high degrees of sequence similarity between UA159 and GS-5, a number of mutants in GS-5 background were constructed by transformation of GS-5 using PCR products derived from the UA159 mutants, which contained the flanking sequences and an antibiotic marker in place of the target gene.

To construct the PlacA::gfp reporter fusion, the plasmid pPMG containing a superfolder GFP protein (sGFP) (30) was engineered on the basis of a cat-fusion plasmid pJL84, for the purpose of integrating exogenous DNA at the phnA-mtlA locus (31). A set of primers, gfp-5'BamHI and gfp-3'Sphl (Table 3), were designed for the amplification of the gfp gene, using plasmid pCM11 (32) as the template. After digestion with restriction enzymes BamHI and Sphl, the $g f p$ fragment was cloned into pJL84 that was treated with the same enzymes to drop out the cat gene, resulting in plasmid pPMG. Subsequently, the lacA promoters from UA159 and GS-5 were amplified using primers PlacA-5Sc and PlacA-3Bm (Table 3) and directionally cloned into Sacl and BamHI sites in pPMG. These plasmids were then used to transform various S. mutans strains for integration of the reporter fusions. All plasmids and genetic mutants engineered in this study were verified by sequencing or PCR, followed by sequencing, respectively, in 
TABLE 3 Primers used in this study

\begin{tabular}{|c|c|c|}
\hline Primer & Sequence $\left(5^{\prime}-3^{\prime}\right)^{a}$ & Purpose \\
\hline manL1000-5 & GCC TTA TCT TTG GAA CAT TTG CCG TT & Deleting manL \\
\hline manMR & TAA TGC TCC CTT CTA GTA ATC CTC TAA & Deleting manL \\
\hline lacG-1 & GTA TTC ATG GTC CTT CTA TTG TTG AG & Deleting lacG \\
\hline lacG-2GA & GCC ATT TAT TAT TTC CTT CCT CTT TTA GCT GCT GTT GCA CCA C & Deleting lacG \\
\hline lacG-3GA & ATA TIT TAC TGG ATG AAT TGT TIT AGT AGA CCA AGA AAA GTG CCT ACT GGT & Deleting lacG \\
\hline lacG-4 & $\overline{\text { GGC TGT TGA CCA AAG AAT AAG GT }}$ & Deleting lacG \\
\hline celA-800up & GCG GCT GCG AGG ATG T & Deleting celA \\
\hline $1456-S$ & AAT GGG CTT CAA ATG TTT TCG & Deleting celB, celR \\
\hline celR-AS & TTC GGG CAT ATT GCT CAA CTC C & Deleting celB \\
\hline celD-AS & GCG ACA AGG AAC CCA TAG TTG CA & Deleting celR \\
\hline lacD-S & TCT TCT CAG ACG AGC GTT TTG G & Deleting lacE \\
\hline lacG-AS & CGA ACACCC TTA ATC TGG & Deleting lacE \\
\hline gfp-5'BamHI & GGA GGA TGA GGA TCC ATG AGC AAA GGA GA & Constructing pPMG \\
\hline gfp-3'Sphl & GGT GGT GGG $\overline{\text { CAT GCT }}$ ATT TGT ACA GCT CAT CCA T & Constructing pPMG \\
\hline PlacA-5'Sc & GAG CAA ATA TAC TGA GCT CAT TAA CTA AAT CGT & PlacA::gfp \\
\hline PlacA-3'Bm & ATT GCC ATG GAT CC $\overline{C T ~ C T C ~ C T T ~ G A A ~ A T G ~ A T T ~}$ & PlacA::gfp \\
\hline glk-1 & GGA TGC CTT GAA TGG TCA TCT & Deleing $g l k$ \\
\hline glk-2 & GCC ATT TAT TAT TTC CTT СCT CTT TTA TGT TCC ACC AAG ATC AAT CCC & Deleing $g l k$ \\
\hline glk-3 & ATA TIT TAC TGG ATG AAT TGT TIT AGT AGA GGG CAG CCA GTC TTG CA & Deleing $g l k$ \\
\hline glk-4 & CGT GGG CAA TGA TAG CAA CAT T & Deleing $g l k$ \\
\hline
\end{tabular}

${ }^{a} \mathrm{An}$ insertion or mutation is indicated by underlining.

order to exclude any clones with unintended mutations in the target genes and in genomic regions immediately adjacent to them.

Biochemical assays. Glucose in the supernates of bacterial cultures were measured using a glucose oxidase kit and a hexokinase kit (MilliporeSigma) according to protocols provided by the supplier. CAT activity (33) and PEP-dependent sugar phosphorylation (PTS) (34) assays were performed according to protocols established in our laboratory, as detailed elsewhere (31).

\section{SUPPLEMENTAL MATERIAL}

Supplemental material for this article may be found at https://doi.org/10.1128/AEM .00864-18.

SUPPLEMENTAL FILE 1, PDF file, 1.8 MB.

\section{ACKNOWLEDGMENT}

This study was supported by grants DE12236 and DE13239 from the National Institute for Dental and Craniofacial Research.

\section{REFERENCES}

1. Carlsson J. 1983. Regulation of sugar metabolism in relation to feastand-famine existence of plaque, p 205-211. In Guggenheim B (ed), Cariology today. Karger, Basel, Switzerland.

2. Deutscher J. 2008. The mechanisms of carbon catabolite repression in bacteria. Curr Opin Microbiol 11:87-93. https://doi.org/10.1016/j.mib .2008.02.007.

3. Colby SM., Russell RRB. 1997. Sugar metabolism by mutans streptococci. J Appl Microbiol 83:80S-88S. https://doi.org/10.1046/j.1365-2672.83.s1 .9.x.

4. Abranches J, Nascimento MM, Zeng L, Browngardt CM, Wen ZT, Rivera MF, Burne RA. 2008. CcpA regulates central metabolism and virulence gene expression in Streptococcus mutans. J Bacteriol 190:2340-2349. https://doi.org/10.1128/JB.01237-07.

5. Zeng L, Choi SC, Danko CG, Siepel A, Stanhope MJ, Burne RA. 2013. Gene regulation by $\mathrm{CcpA}$ and catabolite repression explored by RNA-Seq in Streptococcus mutans. PLoS One 8:e60465. https://doi.org/10.1371/jour nal.pone.0060465.

6. Zeng L, Burne RA. 2008. Multiple sugar: phosphotransferase system permeases participate in catabolite modification of gene expression in Streptococcus mutans. Mol Microbiol 70:197-208. https://doi.org/10 $.1111 /$ j.1365-2958.2008.06403.x.

7. Zeng L, Burne RA. 2009. Transcriptional regulation of the cellobiose operon of Streptococcus mutans. J Bacteriol 191:2153-2162. https://doi .org/10.1128/JB.01641-08.
8. Zeng L, Burne RA. 2010. Seryl-phosphorylated HPr regulates CcpAindependent carbon catabolite repression in conjunction with PTS permeases in Streptococcus mutans. Mol Microbiol 75:1145-1158. https:// doi.org/10.1111/j.1365-2958.2009.07029.x.

9. Zeng L, Das S, Burne RA. 2010. Utilization of lactose and galactose by Streptococcus mutans: transport, toxicity, and carbon catabolite repression. J Bacteriol 192:2434-2444. https://doi.org/10.1128/JB.0162 4-09.

10. Abranches J, Candella MM, Wen ZT, Baker HV, Burne RA. 2006. Different roles of EIIABMan and EIIGlc in regulation of energy metabolism, biofilm development, and competence in Streptococcus mutans. J Bacteriol 188:3748-3756. https://doi.org/10.1128/JB.00169-06.

11. Moye ZD, Zeng L, Burne RA. 2013. Modification of gene expression and virulence traits in Streptococcus mutans in response to carbohydrate availability. Appl Environ Microbiol 80:972-985. https://doi.org/10.1128/ AEM.03579-13.

12. Zeng L, Burne RA. 2013. Comprehensive mutational analysis of sucrosemetabolizing pathways in Streptococcus mutans reveals novel roles for the sucrose phosphotransferase system permease. J Bacteriol 195: 833-843. https://doi.org/10.1128/JB.02042-12.

13. Zeng L, Burne RA. 2015. Sucrose- and fructose-specific effects on the transcriptome of Streptococcus mutans, as determined by RNA sequencing. Appl Environ Microbiol 82:146-156. https://doi.org/10.1128/AEM $.02681-15$ 
14. Zeng L, Chakraborty B, Farivar T, Burne RA. 2017. Coordinated regulation of the EIIMan and fruRKI operons of Streptococcus mutans by global and fructose-specific pathways. Appl Environ Microbiol 83:e01403-17. https:// doi.org/10.1128/AEM.01403-17.

15. Honeyman AL, Curtiss R, III. 1993. Isolation, characterization and nucleotide sequence of the Streptococcus mutans lactose-specific enzyme II (lacE) gene of the PTS and the phospho- $\beta$-galactosidase (lacG) gene. J Gen Microbiol 139:2685-2694. https://doi.org/10.1099/00221287-139-11 -2685 .

16. Liberman ES, Bleiweis AS. 1984. Role of the phosphoenolpyruvatedependent glucose phosphotransferase system of Streptococcus mutans GS5 in the regulation of lactose uptake. Infect Immun 43:536-542.

17. Aleksandrzak-Piekarczyk T, Kok J, Renault P, Bardowski J. 2005. Alternative lactose catabolic pathway in Lactococcus lactis IL1403. Appl Environ Microbiol 71:6060-6069. https://doi.org/10.1128/AEM.71.10.6060-6069 .2005 .

18. Aleksandrzak-Piekarczyk T, Polak J, Jezierska B, Renault P, Bardowski J. 2011. Genetic characterization of the CcpA-dependent, cellobiosespecific PTS system comprising CelB, PtcB, and PtcA that transports lactose in Lactococcus lactis IL1403. Int J Food Microbiol 145:186-194. https://doi.org/10.1016/j.ijfoodmicro.2010.12.011.

19. Fleming E, Camilli A. 2016. ManLMN is a glucose transporter and central metabolic regulator in Streptococcus pneumoniae. Mol Microbiol 102: 467-487. https://doi.org/10.1111/mmi.13473.

20. Sato Y, Okamoto-Shibayama K, Azuma T. 2015. Additional glucose-PTS induction in Streptococcus mutans mutant deficient in mannose- and cellobiose-PTS. Bull Tokyo Dent Coll 56:185-118. https://doi.org/10.2209/ tdcpublication.56.185.

21. Sato Y, Okamoto-Shibayama K, Azuma T. 2015. Glucose-PTS involvement in maltose metabolism by Streptococcus mutans. Bull Tokyo Dent Coll 56:93-103. https://doi.org/10.2209/tdcpublication.56.93.

22. Zeng L, Xue P, Stanhope MJ, Burne RA. 2013. A galactose-specific sugar: phosphotransferase permease is prevalent in the non-core genome of Streptococcus mutans. Mol Oral Microbiol 28:292-301. https://doi.org/10 .1111/omi.12025.

23. Gaca AO, Colomer-Winter C, Lemos JA. 2015. Many means to a common end: the intricacies of (p)ppGpp metabolism and its control of bacterial homeostasis. J Bacteriol 197:1146-1156. https://doi.org/10.1128/JB.0257 7-14.

24. Lemos JA, Lin VK, Nascimento MM, Abranches J, Burne RA. 2007. Three gene products govern (p)ppGpp production by Streptococcus mutans. Mol Microbiol 65:1568-1581. https://doi.org/10.1111/j.1365-2958.2007 .05897.x.

25. Kaspar J, Kim JN, Ahn SJ, Burne RA. 2016. An essential role for (p)ppGpp in the integration of stress tolerance, peptide signaling, and competence development in Streptococcus mutans. Front Microbiol 7:1162. https:// doi.org/10.3389/fmicb.2016.01162.
26. Zeng L, Farivar T, Burne RA. 2016. Amino sugars enhance competitiveness of beneficial commensals with Streptococcus mutans through multiple mechanisms. Appl Environ Microbiol 82:3671-3682. https://doi.org/ 10.1128/AEM.00637-16.

27. Kessler JR, Cobe BL, Richards GR. 2017. Stringent response regulators contribute to recovery from glucose phosphate stress in Escherichia coli. Appl Environ Microbiol https://doi.org/10.1128/AEM.01636-17.

28. Terleckyj B, Willett NP, Shockman GD. 1975. Growth of several cariogenic strains of oral streptococci in a chemically defined medium. Infect Immun 11:649-655.

29. Lau PC, Sung CK, Lee JH, Morrison DA, Cvitkovitch DG. 2002. PCR ligation mutagenesis in transformable streptococci: application and efficiency. J Microbiol Methods 49:193-205. https://doi.org/10.1016/S0167-7012(01) 00369-4.

30. Pedelacq JD, Cabantous S, Tran T, Terwilliger TC, Waldo GS. 2006. Engineering and characterization of a superfolder green fluorescent protein. Nat Biotechnol 24:79-88. https://doi.org/10.1038/nbt1172.

31. Zeng L, Wen ZT, Burne RA. 2006. A novel signal transduction system and feedback loop regulate fructan hydrolase gene expression in Streptococcus mutans. Mol Microbiol 62:187-200. https://doi.org/10.1111/j.1365 -2958.2006.05359.x.

32. Lauderdale KJ, Malone CL, Boles BR, Morcuende J, Horswill AR. 2010. Biofilm dispersal of community-associated methicillin-resistant Staphylococcus aureus on orthopedic implant material. J Orthop Res 28:55-61. https://doi.org/10.1002/jor.20943.

33. Shaw WV. 1975. Chloramphenicol acetyltransferase from chloramphenicolresistant bacteria. Methods Enzymol 43:737-755. https://doi.org/10.1016/ 0076-6879(75)43141-X.

34. LeBlanc DJ, Crow VL, Lee LN, Garon CF. 1979. Influence of the lactose plasmid on the metabolism of galactose by Streptococcus lactis. J Bacteriol 137:878-884.

35. Abranches J, Chen YY, Burne RA. 2003. Characterization of Streptococcus mutans strains deficient in EllABMan of the sugar phosphotransferase system. Appl Environ Microbiol 69:4760-4769. https://doi.org/10.1128/ AEM.69.8.4760-4769.2003.

36. Lemos JA, Brown TA, Jr, Burne RA. 2004. Effects of RelA on key virulence properties of planktonic and biofilm populations of Streptococcus mutans. Infect Immun 72:1431-1440. https://doi.org/10.1128/IAI.72.3.1431 $-1440.2004$

37. Palmer SR, Miller JH, Abranches J, Zeng L, Lefebure T, Richards VP, Lemos JA, Stanhope MJ, Burne RA. 2013. Phenotypic heterogeneity of genomically diverse isolates of Streptococcus mutans. PLoS One 8:e61358. https://doi.org/10.1371/journal.pone.0061358.

38. Sissons $\mathrm{CH}$, Hancock EM, Perinpanayagam HE, Cutress TW. 1988. The bacteria responsible for ureolysis in artificial dental plaque. Arch Oral Biol 33:727-733. https://doi.org/10.1016/0003-9969(88)90006-4. 